\title{
Flotillin-1 expression in human clear-cell renal cell carcinoma is associated with cancer progression and poor patient survival
}

\author{
YIYAN ZHANG, JIJUN LI, YAN SONG, FENGKUN CHEN, YIN PEI and FENGHUA YAO \\ Department of Nephrology, First Affiliated Hospital of Chinese PLA General Hospital, Beijing 100048, P.R. China
}

Received October 22, 2013; Accepted May 19, 2014

DOI: $10.3892 / \mathrm{mmr} .2014 .2310$

\begin{abstract}
The present study was designed to elucidate the expression levels and the proliferative effect of flotillin-1, an integral membrane protein encoded by the FLOT1 gene, in human clear-cell renal cell carcinoma (RCC). Flotillin has been implicated in other types of cancer, but the role of flotillin in RCC has not been established. Immunohistochemistry and western blotting were used to determine FLOT1 protein expression levels in RCC samples from 182 patients who underwent nephrectomy. FLOT1 mRNA expression levels were analyzed using reverse-transcription (RT) and RT-quantitative polymerase chain reaction (PCR). The association between FLOT1 expression levels in the tumor samples and patient survival time was examined using Kaplan-Meier analysis. To demonstrate the proliferative effect of FLOT1 on RCC cells, a FLOT1 vector was transfected into four RCC cell lines and FLOT1 expression was inhibited using small interfering RNA. The proliferative ability of the RCC cells was investigated using a WST-1 assay and xenograft experiments with BALB/C nude mice. The results demonstrated that FLOT1 expression levels were significantly higher in RCC cell samples from patients than in healthy renal tissue, and the expression levels were associated with tumor stage, size and histological grade. In addition, FLOT1 significantly enhanced the proliferation of RCC cell lines in vitro and in vivo. These findings suggest that FLOT1, which is upregulated in RCC, is involved in RCC cell proliferation, tumorigenesis and progression. Therefore, FLOT1 is an independent prognostic marker and therapeutic target for patients with clear-cell RCC.
\end{abstract}

\section{Introduction}

Human renal cell carcinoma (RCC) is the most common and lethal type of malignant kidney tumor. Clear-cell renal cell carcinoma is the most common type of RCC, constituting

Correspondence to: Mr Jijun Li, Department of Nephrology, First Affiliated Hospital of Chinese PLA General Hospital, 51 Fucheng Road, Beijing 100048, P.R. China

E-mail: jijunli89@163.com

Key words: renal cell carcinoma, flotillin-1, prognosis
$85 \%$ of cases (1). At the localized stage, RCC is considered resectable; however, $30 \%$ patients with limited disease at surgery experience metastasis within three years (2). Once RCC metastasizes, the prognosis is poor (3). Therapeutic options for advanced RCC are limited since RCC is resistant to conventional chemotherapy $(4,5)$. Novel treatment options include immunotherapy (6), monoclonal antibody treatment (7), targeted treatment (8) and inhibition of signal transductions (9). Recently, molecular markers have been evaluated in terms of their prognostic predictive ability and potential as therapeutic targets for RCC $(10,11)$; however, the molecular mechanisms underlying RCC progression and development remain unclear.

The dynamic structure of the cell membrane is important in a number of biological processes, such as cell growth, cell migration, cell survival and metastasis. Glycolipoprotein microdomains, or lipid rafts, serve as physical platforms for various molecules to coordinate a variety of signal-transduction pathways (12-14). As lipid rafts have been reported to be involved in the initiation and development of numerous types of cancer $(15,16)$, lipid rafts may be novel therapeutic targets against malignant tumors $(17,18)$.

The flotillin protein family (also known as the reggie family) contains two homologous isoforms, FLOT1 and FLOT2, which are essential markers of lipid rafts (19-21). FLOT1 and FLOT2 interact to form a complex that is important in biological processes, such as membrane receptor signaling, membrane trafficking, cell motility and adhesion (22). FLOT1 and FLOT2 also activate signal transductions by binding with membrane-resident receptor kinases. Insulin stimulation may enhance FLOT1-Cbl-Cbl-associated protein complex formation, which generates a signal that is important for the uptake of glucose in adipocytes (23).

Increased Fyn kinase activation has been reported to result in flotillin translocation from the plasma membrane to intracellular organelles and thus involvement in tyrosine kinase-regulated endocytosis (24). In addition to FLOT1 and FLOT2 functions in cellular and organelle membranes, flotillins are also involved in tumorigenesis and the progression of different types of human tumor $(25,26)$. Overexpression of FLOT1 increases the activity of the Aurora B kinase and results in incorrect attachment of microtubules to kinetochores, which suggests that FLOT1 is associated with genomic instability (27). Furthermore, FLOT1 expression has been observed to be upregulated in cancer types originating in epithelial cells, such as colorectal and esophageal squamous 

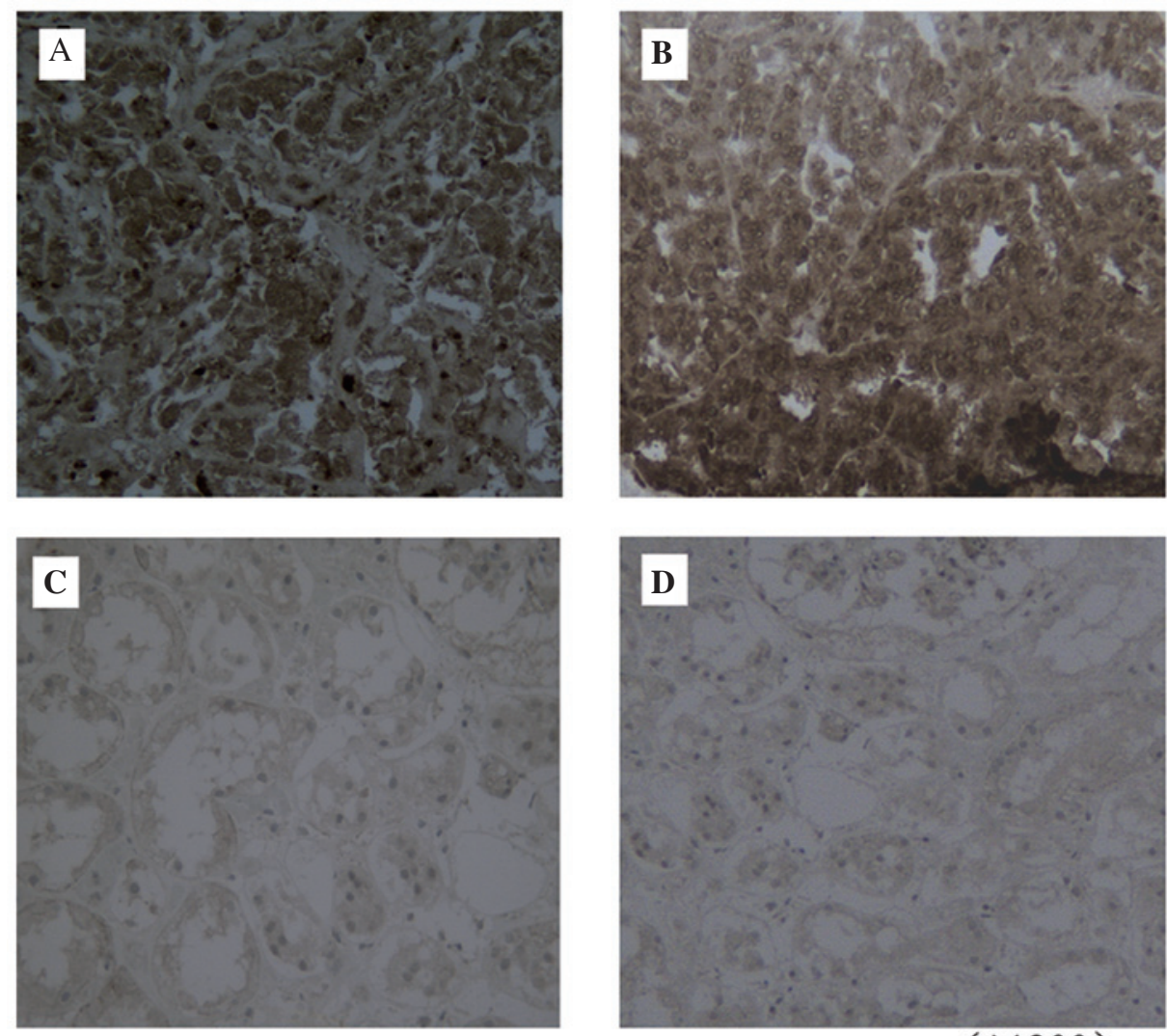

$(\times 200)$

Figure 1. Immunostaining of FLOT1 reveals (A and B) high FLOT1 expression levels in clear-cell renal cell carcinoma tissue but (C and D) negative or low FLOT1 expression levels in corresponding normal healthy renal tissue. FLOT1, flotillin-1 (magnification, x200).

cell cancer $(28,29)$. In another study, overexpression of FLOT1 was reported to enhance the proliferation of $\mathrm{PC}-3$ prostate cancer cells (30). All findings suggest that FLOT1 may be critical in the progression of malignant tumors. In the present study, the expression levels of FLOT1 and its effect on the proliferative ability of human RCC cells were investigated.

\section{Materials and methods}

Patients and tissue samples. This study was approved by the ethics committee of the First Affiliated Hospital of Chinese PLA General Hospital (Beijing, China). A total of 182 patients who had undergone radical surgical treatment for RCC at the Department of Urology, First Affiliated Hospital of Chinese PLA General Hospital were recruited between 1998 and 2003. All patients were informed of the content and agreed to join in the present study. All tumors were determined to be conventional clear-cell RCC by experienced pathologists who were blinded to the patient data. Tumor characteristics and stage were classified using the tumor-node-metastasis (TNM) classification system, and the nuclear grade was assessed according to the Fuhrman tumor grading system $(31,32)$. The RCC samples and corresponding normal healthy renal tissue samples, located as far as possible from the tumor site, were surgically removed, fixed in formalin, dehydrated and paraffin-embedded. All samples were frozen in liquid nitrogen immediately following surgical resection and maintained at $-90^{\circ} \mathrm{C}$ for protein and RNA extraction. Postoperative patients with RCC underwent routine checkups and follow-ups at the outpatient clinic in our hospital for 10 years. Blood biochemistry tests, ultrasonography, magnetic resonance imaging or computed tomography were performed during the follow up.

Cell culture. Four RCC cell lines, ACHN, Caki-1, NC 65 and A498 cells, were obtained from the American Type Culture Collection (Rockville, MD, USA) and cultured in complete medium consisting of RPMI-1640 medium (Gibco, Bio-Cult Diagnostics Limited, Glasgow, UK), 2 mM L-glutamine, $25 \mathrm{mM}$ HEPES, 10\% heat-inactivated fetal bovine serum, 100 units/ml penicillin, $100 \mu \mathrm{g} / \mathrm{ml}$ streptomycin and $1 \%$ nonessential amino acids purchased from Invitrogen Life Technologies (Carlsbad, CA, USA). All cell lines were maintained as monolayers in a $10-\mathrm{cm}$ plastic dish and incubated in a humidified atmosphere containing $5 \% \mathrm{CO}_{2}$ at $37^{\circ} \mathrm{C}$.

RCC xenograft. BALB/C nude mice, aged 3 or 4 weeks, were purchased from CAMS Laboratories (Beijing, China) and randomly divided into control and FLOT1 vector groups containing 15 mice each. A total of $2 \times 10^{7} \mathrm{RCC}$ cells was injected into the back of each mouse. All mice were observed and tumor volumes were recorded once a week. After five weeks, all mice were sacrificed by decapitation under deep anesthesia and the final volume of each tumor was measured.

WST-1 assay. Exponentially growing RCC cells were harvested, seeded in a 96-well microtiter plate (Corning Inc., 
Table I. Characteristics of patients with renal cell carcinoma and FLOT1 expression levels detected using reverse transcription-quantitative polymerase chain reaction and immunohistochemistry.

\begin{tabular}{|c|c|c|c|c|c|c|c|c|}
\hline \multirow[b]{2}{*}{ Variable } & \multirow[b]{2}{*}{$\mathrm{n}$} & \multirow{2}{*}{$\begin{array}{c}\text { FLOT1 mRNA } \\
(\text { mean } \pm \text { SD })\end{array}$} & \multirow[b]{2}{*}{ P-value } & \multicolumn{4}{|c|}{ FLOT1 protein } & \multirow[b]{2}{*}{ P-value } \\
\hline & & & & - & + & ++ & +++ & \\
\hline Renal cell carcinoma & 182 & $1.53 \pm 0.26$ & & 19 & 84 & 48 & 31 & \\
\hline Healthy kidney & 182 & $0.41 \pm 0.12$ & $<0.05$ & 153 & 21 & 8 & 0 & $<0.05$ \\
\hline \multicolumn{9}{|l|}{ Gender } \\
\hline Male & 99 & $1.51 \pm 0.28$ & & 11 & 46 & 26 & 16 & \\
\hline Female & 83 & $1.55 \pm 0.24$ & $>0.05$ & 8 & 38 & 22 & 15 & $>0.05$ \\
\hline \multicolumn{9}{|l|}{ Age (years) } \\
\hline$<60$ & 101 & $1.53 \pm 0.22$ & & 10 & 46 & 28 & 17 & \\
\hline$\geq 60$ & 81 & $1.52 \pm 0.26$ & $>0.05$ & 9 & 38 & 20 & 14 & $>0.05$ \\
\hline \multicolumn{9}{|l|}{ Tumor size (cm) } \\
\hline$\leq 7$ & 98 & $1.02 \pm 0.29$ & & 15 & 69 & 9 & 5 & \\
\hline$>7$ & 84 & $2.12 \pm 0.35$ & $<0.05$ & 4 & 15 & 39 & 26 & $<0.05$ \\
\hline \multicolumn{9}{|l|}{ Histologic grade $(G)$} \\
\hline G1 & 97 & $0.81 \pm 0.18$ & & 15 & 65 & 14 & 3 & \\
\hline $\mathrm{G} 2$ & 60 & $1.71 \pm 0.27$ & & 4 & 19 & 31 & 6 & \\
\hline G3 & 25 & $3.92 \pm 0.36$ & $<0.05$ & 0 & 0 & 3 & 22 & $<0.05$ \\
\hline \multicolumn{9}{|l|}{ Tumor stage } \\
\hline I & 98 & $1.02 \pm 0.29$ & & 17 & 69 & 9 & 3 & \\
\hline II & 47 & $1.53 \pm 0.25$ & & 2 & 15 & 26 & 4 & \\
\hline III & 23 & $2.49 \pm 0.38$ & & 0 & 0 & 11 & 12 & \\
\hline IV & 14 & $3.54 \pm 0.42$ & $<0.05$ & 0 & 0 & 2 & 12 & $<0.05$ \\
\hline
\end{tabular}

FLOT1, flotillin-1; SD, standard deviation.

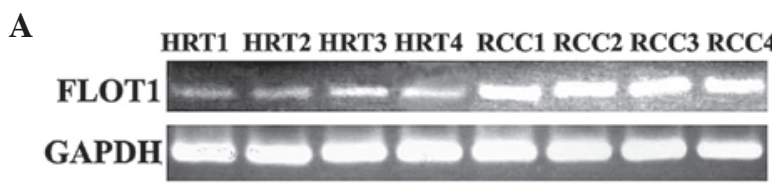

B HRT1 HRT2 HRT3 HRT4 RCC1 RCC2 RCC3 RCC4

(RT-PCR)

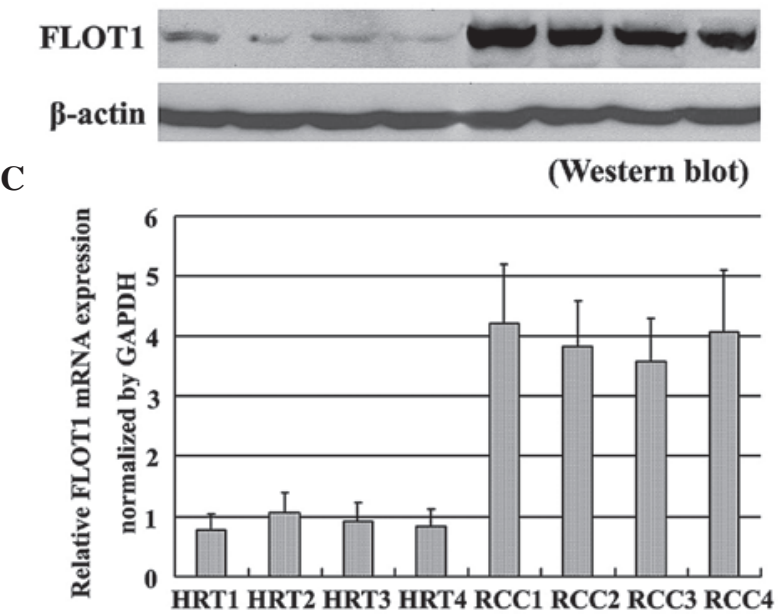

(RT-qPCR)

Figure 2. FLOT1 expression in RCC and HRT was confirmed by (A) RT-PCR, (B) western blotting and (C) RT-qPCR in all samples. The results from four pairs of samples are shown. All experiments were performed in triplicate and error bars indicate standard deviation. FLOT1, flotillin-1; PCR, polymerase chain reaction; RT-PCT, reverse transcription PCR; RT-qPCR, reverse transcription-quantitative PCR RCC, renal cell carcinoma, HRT, healthy renal tissue. 
A
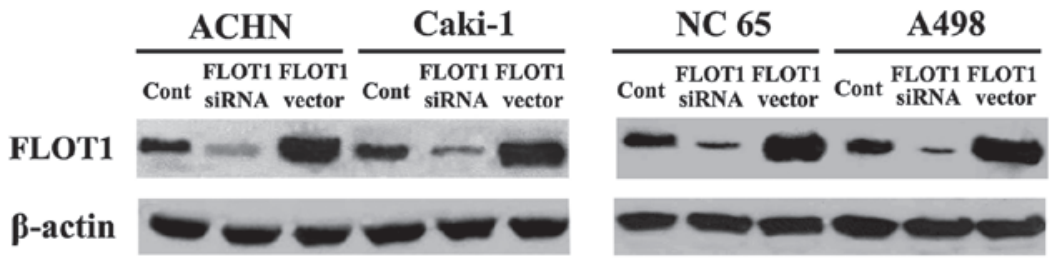

B

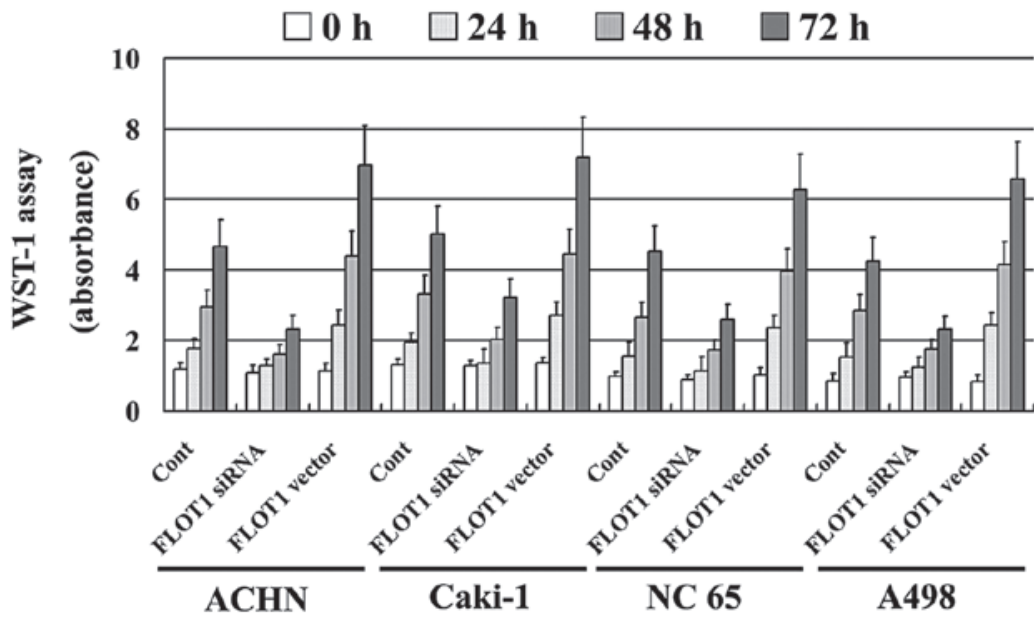

C

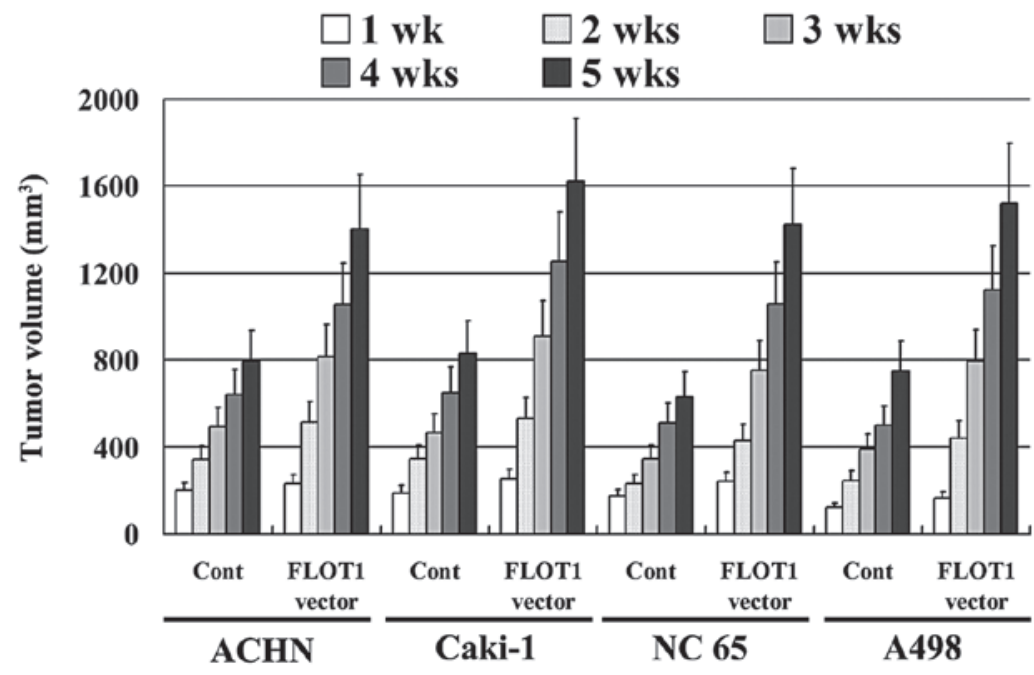

Figure 3. Effect of FLOT1 on the proliferation of four renal cell carcinoma cell lines (ACHN, Caki-1, NC 65 and A498). In the treatment groups, the FLOT1 expression vector was stably transfected into the cells. FLOT1 expression levels were reduced using siRNA. (A) All transfections were confirmed by western blotting. The proliferative ability of renal carcinoma cells was examined using (B) in vitro WST-1 assay and (C) an in vivo xenograft experiment with BALB/C nude mice. FLOT1, flotillin-1; siRNA, small interfering RNA; Cont, control.

Corning, NY, USA) and placed in an incubator. At 24, 48 and 72 h, $10 \mu \mathrm{l}$ WST-1 (Roche Diagnostics GmbH, Penzberg, Germany) was added to each well and the incubation was continued for an additional $2 \mathrm{~h}$. The absorbance, reflected in the cell count in each well, was measured with a microculture plate reader (Immunoreader; Japan Intermed Co. Ltd., Tokyo, Japan) at $450 \mathrm{~nm}$.

Immunohistochemistry. Paraffin sections (5 $\mu \mathrm{m})$ were deparaffinized in xylene and rehydrated using graded alcohol. Endogenous peroxidase activity was inhibited with administration of $0.3 \%$ hydrogen peroxide for $15 \mathrm{~min}$. The sections were blocked with $20 \%$ normal rabbit serum (Sigma, St. Louis, MO, USA) for $30 \mathrm{~min}$ prior to 1 -h incubation with primary FLOT1 rabbit monoclonal antibody (Cell Signaling Technology,
Inc., Danvers, MA, USA). The slides were washed twice in Tris-buffered saline and incubated with biotinylated rabbit anti-rabbit antibody (Dako, Glostrup, Denmark). Detection of the antibody reaction was performed using a standard streptavidin-biotin complex (Sigma). The immunohistochemistry results were obtained using a light microscope (Olympus BH2; Olympus, Tokyo, Japan) and FLOT1 immunostaining was conducted to semiquantitatively evaluate the slides for intensity (-, negative; + , weak; ++ , moderate and +++ , strong staining).

Reverse transcription $(R T)$ and RT-quantitative polymerase chain reaction $(R T-q P C R)$. mRNA was isolated from normal and RCC tissue using the QuickPrep mRNA purification kit (GE Healthcare, Little Chalfont, UK) according to the 


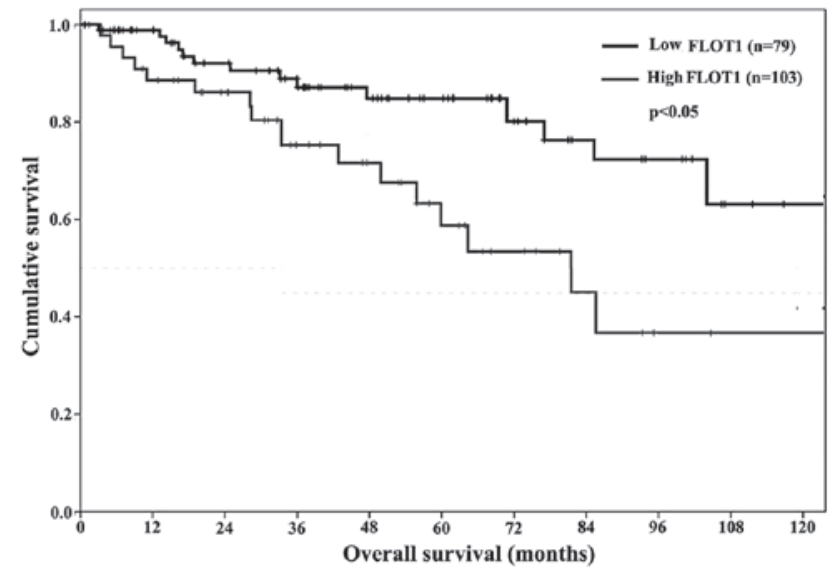

Figure 4. Kaplan-Meier analysis was used to examine the correlation of FLOT1 expression levels and the survival times of patients with renal cell carcinoma. High FLOT1 expression levels were associated with poor prognosis. FLOT1, flotillin-1.

manufacturer's instructions. A first-strand cDNA synthesis kit (Amersham Biosciences, Amersham, UK) was used for reverse transcription. The PCR conditions were determined according to the manufacturer's instructions and the PCR product was confirmed using agarose gel electrophoresis. qPCR was conducted with the LC FastStart DNA Master SYBR Green I (Roche Applied Science, Penzberg, Germany) and the products were quantified with a LightCycler (Roche Applied Science). The primers used were as follows: forward: 5'-CCCATCTCAGTCACTGGCATT-3' and reverse: 5'-CCGCCAACATCTCCTTGTTC-3' for FLOT1; and forward: 5'-ATCAAGAAGGTGGTGAAGCAGG-3' and reverse: 5'-GTGGAGGAGTGGGTGTCGC-3' for GAPDH.

Western blotting. The protein was extracted and protein concentration assessed according to the manufacturer's instructions, and SDS polyacrylamide gel electrophoresis was performed. FLOT1 antibody (dilution 1:1,000) and secondary HRP conjugated antibody (dilution 1:2,000) were was purchased from Cell Signaling Technology, Inc.. Anti- $\beta$-actin monoclonal antibody (Abcam, Cambridge, UK) served as a loading control. The immune complexes were examined using electrochemiluminescence (Amersham, Aylesbury, UK).

Small interfering (si)RNA and transfection. siRNA oligonucleotide sequences were designed with siDirect software (University of Tokyo, Tokyo, Japan). RCC cells were seeded in plastic culture dishes in complete medium without antibiotics until 40 to $50 \%$ confluence was achieved. The cells were then transfected with siRNA oligonucleotides using Lipofectamine 2000 (Invitrogen Life Technologies). After $48 \mathrm{~h}$ incubation, gene expression was confirmed with western blotting. The coding sequence of human FLOT1 was cloned using RT-PCR with HK-2 normal kidney cell line (purchased from ATCC, Manassas, VA, USA) cDNA as a substrate, and the products were subcloned into pcDEF3, a mammalian expression vector (Invitrogen Life Technologies). The RCC cell lines were stably transfected with Lipofectamine 2000. Using this expression vector, which contained full-length FLOT1 cDNA, monoclonal antibodies were selected using G418 aminoglycoside antibiotics (Calbiochem, Bad Soden, Germany) and gene expression was confirmed by western blotting.

Statistical analysis. SPSS version 13.0 (SPSS, Inc., Chicago, IL, USA) was used to perform statistical calculations. All experiments were performed in triplicate and the results expressed as the mean \pm standard deviation. Statistical significance was determined using Student's t-test, and the $\chi^{2}$ test was used to evaluate the association between FLOT1 expression levels and clinicopathological parameters. Survival curves were plotted using Kaplan-Meier analysis. $\mathrm{P} \leq 0.05$ was considered to indicate a statistically significant difference.

\section{Results}

Patient characteristics. Subjects comprised 99 male and 83 female patients, aged 42-86 years (mean age, 64 years). The RCC tumor sizes were between 2 and $13 \mathrm{~cm}$ (average, $4.5 \mathrm{~cm})$. A total of 98 patients had stage I cancer, $47 \mathrm{had}$ stage II, 23 had stage III and 14 had stage IV. With regards to Fuhrman tumor grade, 97 patients had grade 1, 60 had grade 2 and 25 had grade 3 . The presenting symptoms included flank pain (21 patients), hematuria (19 patients) and detection of a palpable mass (15 patients).

Malignancy was an incidental finding on routine examination in 98 patients. Laboratory examination at diagnosis revealed an elevated sedimentation rate in 32 patients, while anemia, thrombocytopenia and erythrocytosis were each identified in two patients. A total of 37 patients suffered from one or more comorbidities, including diabetes mellitus, urolithiasis, angina pectoris and valvular heart disease. Five patients had previously been treated with a radical nephrectomy on the other side and 12 patients exhibited metastatic disease at diagnosis.

FLOT1 protein expression levels in RCC. FLOT1 protein expression levels in human RCC and normal healthy renal tissue were determined using immunohistochemistry. FLOT1 expression was upregulated in RCC tissue compared with in the corresponding normal renal tissue (Fig. 1). FLOT1 expression was observed in 163 of $182 \mathrm{RCC}$ tumors $(89.6 \%)$ but in only 29 of $182(15.9 \%)$ normal renal tissue samples. $\chi^{2}$ analysis revealed significant associations between increased FLOT1 protein expression levels and advanced tumor stage, high histological grade and large tumor size (all $\mathrm{P}<0.05)$. However, no significant differences were detected between gender and age variables and FLOT1 protein expression levels (Table I). The results from this sample analysis suggest that FLOT1 is involved in tumorigenesis and RCC progression.

FLOT1 expression levels analyzed by RT-qPCR and western blotting. To confirm the results demonstrating upregulated FLOT1 expression in RCC through immunohistochemical analysis, RT-qPCR and western blotting were used to measure FLOT1 expression levels in human RCC and normal healthy renal tissue. The levels of FLOT1 expression were determined relative to an internal control. The results indicated that FLOT1 expression was upregulated to a significantly greater extent in RCC than in the corresponding normal renal tissue $(\mathrm{P}=0.001)$ and that FLOT1 expression levels were similar to 
those detected using immunohistochemistry in all samples. The results of four pairs of samples are shown in Fig. 2.

Effect of FLOT1 on RCC cell proliferation. An expression vector containing full-length FLOT1 cDNA was stably transfected into ACHN, Caki-1, NC 65 and A498 cell lines, and siRNA was used to inhibit FLOT1 expression. All transfections were confirmed by western blotting. FLOT1 expression was significantly downregulated by siRNA and upregulated by the FLOT1 vector insertion (Fig. 3A). The effect of FLOT1 on RCC cell proliferative ability in vitro was analyzed using a WST-1 assay, which revealed that RCC cells expressing high levels of FLOT1 exhibited significantly higher proliferative ability than control cells $(\mathrm{P}=0.001)$. By contrast, $\mathrm{RCC}$ cells with low FLOT1 expression levels, due to siRNA administration, exhibited lower proliferative ability $(\mathrm{P}=0.002$; Fig. 3B). Similar results were observed in vivo in the xenograft experiment with BALB/C nude mice ( $\mathrm{P}=0.001$; Fig. 3C).

Prognostic significance of FLOT1 expression levels. As FLOT1 expression levels, and RCC stage and grade were correlated, the possibilities for FLOT1 as a prognostic marker in human RCC were investigated. Kaplan-Meier analysis was used to calculate the association between the FLOT1 expression levels detected by immunohistochemistry (classified as low, - or +; or high, ++ or +++ ) and patient survival times. A total of nine patients succumbed to myocardial infarction and five patients succumbed to disseminated malignant disease. The average survival time was significantly greater in the group with low FLOT1 expression levels compared with the group with high FLOT1 expression levels $(\mathrm{P}<0.05$; Fig. 4). After 10 years follow-up, 51 of $79(64.6 \%)$ patients with low FLOT1 expression levels were alive and disease-free, compared with 35 of 103 (34\%) patients with high FLOT1 expression levels. Therefore, FLOT1 expression levels were an independent prognostic marker.

\section{Discussion}

In recent years, FLOT1 has received considerable attention regarding its involvement in tumorigenesis; FLOT1 expression has been observed in different types of human tumor. The present study found that FLOT1 expression was increased in patients with RCC compared with normal individuals. Pathological analysis revealed that FLOT1 was expressed in 163 out of 182 RCC patients (89.6\%) compared with 29 out of $182(15.9 \%)$ in the controls. This result was further confirmed using RT-PCR, western blot analysis and RT-qPCR. These results demonstrated a correlation between FLOT1 expression and RCC. One study demonstrated that FLOT1 expression levels are associated with aggressive hepatocellular carcinoma and may be used as a prognostic marker in patients with this disease (33). Another study indicated that FLOT1 regulates ErbB2 activation in breast cancer (34) and that silencing FLOT1 expression significantly suppresses the proliferation of breast cancer cells in vitro and in vivo (35). A further study suggested that FLOT1 enhanced cell proliferation and invasive ability by activating the nuclear factor- $\kappa \mathrm{B}$ signaling pathway in esophageal squamous cell carcinoma cells (25). In the present study, statistical analysis of the data demonstrated a high degree of association between increased FLOT1 expression and advanced tumor stage, high histological grade and large tumor size. Using FLOT1 siRNA transfection it was also demonstrated that the absence of FLOT1 in renal carcinoma cell lines led to a significant decrease in cell proliferation compared with cells transfected with the FLOT1 vector. These results demonstrated that FLOT1 expression may be essential in tumor growth and progression. Thus, high FLOT1 expression levels may be considered a valuable marker for prediction of poor prognosis of patients with esophageal squamous cell carcinoma and breast cancer (33). A Kaplan-Meier analysis of our data on the correlation between FLOT1 expression and long term survival revealed that patients with low FLOT1 expression had a higher disease free survival rate, 51 out of 79 $(64.6 \%)$ patients after 10 years, compared with 35 of $103(34 \%)$ patients with high FLOT1 expression. This demonstrated that FLOT1 expression could be an independent prognostic marker. These findings suggest that FLOT1 exhibits an oncogenic role in human tumors, including in PC-3 prostate cancer (30). prostate cancer. However, to the best of our knowledge, this is the first study regarding FLOT1 expression levels in human RCC.

In the present study, RCC patients with high levels of FLOT1 expression were found to have poor survival times, thus FLOT1 may be a useful prognostic marker and valuable in follow-up monitoring of patients with RCC. Furthermore, silencing FLOT1 expression may prove to be a novel treatment strategy in the future. An investigation into the detailed molecular mechanisms of FLOT1 in clear-cell RCC is indicated.

\section{References}

1. Deng FM and Melamed J: Histologic variants of renal cell carcinoma: does tumor type influence outcome? Urol Clin North Am 39: 119-132, 2012.

2. Athar U and Gentile TC: Treatment options for metastatic renal cell carcinoma: a review. Can J Urol 15: 3954-3966, 2008.

3. Jemal A, Siegel R, Ward E, et al: Cancer statistics, 2006. CA Cancer J Clin 56: 106-130, 2006.

4. Yu DS, Chang SY and Ma CP: The expression of MDR-1-related gp-170 and its correlation with anthracycline resistance in renal cell carcinoma cell lines and multidrug-resistant sublines. Br J Urol 82: 544-547, 1998.

5. Hartmann JT and Bokemeyer C: Chemotherapy for renal cell carcinoma. Anticancer Res 19: 1541-1543, 1999.

6. Coppin C: Immunotherapy for renal cell cancer in the era of targeted therapy. Expert Rev Anticancer Ther 8: 907-919, 2008.

7. Dalle S, Thieblemont C, Thomas L and Dumontet C: Monoclonal antibodies in clinical oncology. Anticancer Agents Med Chem 8: 523-532, 2008

8. Sciarra A, Gentile V, Salciccia S, Alfarone A and Di Silverio F: New anti-angiogenic targeted therapy in advanced renal cell carcinoma (RCC): current status and future prospects. Rev Recent Clin Trials 3: 97-103, 2008.

9. Simpson D and Curran MP: Temsirolimus: in advanced renal cell carcinoma. Drugs 68: 631-638, 2008.

10. Lam JS, Pantuck AJ, Belldegrun AS and Figlin RA: Protein expression profiles in renal cell carcinoma: staging, prognosis, and patient selection for clinical trials. Clin Cancer Res 13: 703 s-708s, 2007.

11. Netto GJ and Cheng L: Emerging critical role of molecular testing in diagnostic genitourinary pathology. Arch Pathol Lab Med 136: 372-390, 2012.

12. Lingwood D and Simons K: Lipid rafts as a membrane-organizing principle. Science 327: 46-50, 2010.

13. Jacobson K, Mouritsen OG and Anderson RG: Lipid rafts: at a crossroad between cell biology and physics. Nat Cell Biol 9: 7-14, 2007.

14. Simons K and Toomre D: Lipid rafts and signal transduction. Nat Rev Mol Cell Biol 1: 31-39, 2000.

15. Patra SK: Dissecting lipid raft facilitated cell signaling pathways in cancer. Biochim Biophys Acta 1785: 182-206, 2008. 
16. Patra SK and Bettuzzi S: Epigenetic DNA-methylation regulation of genes coding for lipid raft-associated components: a role for raft proteins in cell transformation and cancer progression (review). Oncol Rep 17: 1279-1290, 2007.

17. Mollinedo F, de la Iglesia-Vicente J, Gajate, C, et al: Lipid raft-targeted therapy in multiple myeloma. Oncogene 29: $3748-3757,2010$

18. Hitosugi T, Sato M, Sasaki K and Umezawa Y: Lipid raft specific knockdown of SRC family kinase activity inhibits cell adhesion and cell cycle progression of breast cancer cells. Cancer Res 67: 8139-8148, 2007.

19. Lang DM, Lommel S, Jung M, et al: Identification of reggie-1 and reggie-2 as plasmamembrane-associated proteins which cocluster with activated GPI-anchored cell adhesion molecules in noncaveolar micropatches in neurons. J Neurobiol 37: 502-523, 1998.

20. Stuermer CA, Lang DM, Kirsch F, et al: Glycosylphosphatidyl inositol-anchored proteins and fyn kinase assemble in noncaveolar plasma membrane microdomains defined by reggie- 1 and -2 . Mol Biol Cell 12: 3031-3045, 2001

21. Schulte T, Paschke KA, Laessing U, Lottspeich F and Stuermer CA: Reggie-1 and reggie-2, two cell surface proteins expressed by retinal ganglion cells during axon regeneration. Development 124 577-587, 1997.

22. Banning A, Tomasovic A and Tikkanen R: Functional aspects of membrane association of reggie/flotillin proteins. Curr Protein Pept Sci 12: 725-735, 2011.

23. Baumann CA, Ribon V, Kanzaki M, et al: CAP defines a second signaling pathway required for insulin-stimulated glucose transport. Nature 407: 202-207, 2000.

24. Riento K, Frick M, Schafer I and Nichols BJ: Endocytosis of flotillin-1 and flotillin-2 is regulated by Fyn kinase. J Cell Sci 122: 912-918, 2009.

25. Song L, Gong H, Lin C, et al: Flotillin-1 promotes tumor necrosis factor-alpha receptor signaling and activation of NF-kappaB in esophageal squamous cell carcinoma cells. Gastroenterology 143 : 995-1005, 2012.
26. Hazarika P, McCarty MF, Prieto VG, et al: Up-regulation of Flotillin-2 is associated with melanoma progression and modulates expression of the thrombin receptor protease activated receptor 1. Cancer Res 64: 7361-7369, 2004.

27. Santamaría A, Castellanos E, Gómez V, et al: PTOV1 enables the nuclear translocation and mitogenic activity of flotillin-1, a major protein of lipid rafts. Mol Cell Biol 25: 1900-1911, 2005 .

28. Thorn CC, Freeman TC, Scott N, Guillou PJ and Jayne DG: Laser microdissection expression profiling of marginal edges of colorectal tumours reveals evidence of increased lactate metabolism in the aggressive phenotype. Gut 58: 404-412, 2009.

29. Lin $\mathrm{C}, \mathrm{Wu} \mathrm{Z}$, Lin $\mathrm{X}$, et al: Knockdown of FLOT1 impairs cell proliferation and tumorigenicity in breast cancer through upregulation of FOXO3a. Clin Cancer Res 17: 3089-3099, 2011.

30. Gómez V, Sesé M, Santamaría A, et al: Regulation of aurora B kinase by the lipid raft protein flotillin-1. J Biol Chem 285: 20683-20690, 2010

31. Fuhrman SA, Lasky LC and Limas C: Prognostic significance of morphologic parameters in renal cell carcinoma. Am J Surg Pathol 6: 655-663, 1982

32. Delahunt B, Sika-Paotonu D, Bethwaite PB, William Jordan T, Magi-Galluzzi C, Zhou M, Samaratunga H and Srigley JR: Grading of clear cell renal cell carcinoma should be based on nucleolar prominence. Am J Surg Pathol 35: 1134-1139, 2011.

33. Zhang SH, Wang CJ, Shi L, et al: High expression of FLOT1 is associated with progression and poor prognosis in hepatocellular carcinoma. PLoS ONE 8: e64709, 2013.

34. Pust S, Klokk TI, Musa N, et al: Flotillins as regulators of ErbB2 levels in breast cancer. Oncogene 32: 3443-3451, 2013.

35. Lin $\mathrm{C}, \mathrm{Wu} \mathrm{Z}$, Lin $\mathrm{X}$, et al: Knockdown of FLOT1 impairs cell proliferation and tumorigenicity in breast cancer through upregulation of FOXO3a. Clin Cancer Res 17: 3089-3099, 2011. 